\title{
An Efficient Analytical Solution of Blast Wave Problem in Real Gas Flow under the Influence of Dust-Laden Particles
}

\author{
Akmal Husain, V. K. Singh, Syed Aftab Haider
}

\begin{abstract}
In the present paper, we investigated the problem of the propagation of blast waves governed by a nonhomogeneous quasilinear hyperbolic system of partial differential equations (PDEs), describing one-dimensional unsteady motion with generalized geometries in a real gas flow (van der Waals gas) in which the influence of the dust particles is significant. An efficient analytical approach has been used to the governing hyperbolic system with respect to the Rankine-Hugoniot $(\mathrm{RH})$ conditions to obtain an exact solution in terms of flow parameters density, velocity and the pressure, which exhibits space-time dependence. Further, an analytical expression for the total energy influenced by real gas effects (consisting of non-ideal gas and small solid dust-laden particles) is derived. The results obtained significantly explore the effect of dust-laden particles on the propagation of blast waves in a van der Waals gas.
\end{abstract}

Keywords: Blast waves, Conservation laws, Dust-laden particles, Gas-dynamics, Rankine-Hugoniot jump conditions, Van der Waals gas

\section{INTRODUCTION}

A blast wave is a kind of strong intermittent compression wave usually generated in gaseous media due to the sudden deposition (e.g. nuclear explosions, supernova explosions and chemical explosions) of large amounts of energy in relatively small regions. Basically, the blast wave is a type of moving shock wave that is inspired by a blast. The study of blast wave propagation in diverse branches of continuum mechanics has received immense importance in the past decades, because it was greatly inspired by many industrial applications from both mathematical and physical perspectives. Besides its industrial applications, it has been widely used with great success to study various problems in plasma physics, astrophysics and non- linear acoustics.

Several approaches have been developed by many investigators in order to gain a better understanding of the

Revised Manuscript Received on February 22, 2020.

* Correspondence Author

Akmal Husain*, Department of Mathematics, SoE University of Petroleum and Energy Studies (UPES), Dehradun, India.

Email: ahusain@ddn.upes.ac.in

V. K Singh, Department of Applied Sciences, Institute of Engineering and Technology, Lucknow, India. Email: singhvijaikrishna9@gmail.com

Syed Aftab Haider, Department of Mathematics, Shia P. G. College, Lucknow, India. Email: aftabhaidershiapgc2019@gmail.com

(C) The Authors. Published by Blue Eyes Intelligence Engineering and Sciences Publication (BEIESP). This is an open access article under the CC BY-NC-ND license (http://creativecommons.org/licenses/by-nc-nd/4.0/) blast wave phenomenon. A detailed mathematical approach to study the propagation of blast waves within the context of hyperbolic system have been proposed by classical work of Taylor [1-2], Sakurai [3-4] and Rogers [5]. Taylor [1-2] developed self-similar solutions for spherical blast waves in his classical theory of blast wave propagation. Sakurai [3-4] used principle of similarity, and gave a power series solution for shock waves of moderate strength. Rogers [5] obtained an analytical solution for the blast wave problem through a medium of varying density for the case of spherically symmetric flows. Bach and Lee [6] reported an approximate solution for the entire range of the blast wave evolution. Poslavskii [7] obtained an exact similarity solution of unsteady one-dimensional strong shock waves in a moving medium. Sharma, Ram and Sachdev [8] pointed out a uniformly valid analytical solution to the problem of interaction of a shock wave with a simple wave and classify the effects of decaying shock on the entire flow profile. Zhuang, Xue, Chen and Jiang [9] examined the physical phenomenon of a high-pressure dense gas explosion, and their organized numerical study showed the development process of Richmond-Meshkov instability and its re-shock phenomenon in detail. In recent years, many attempts have been made in evaluating the analytical solution of blast wave propagation in different material media. Oliveri and Speciale [10-11] have investigated in details an exact solution for the unsteady equation of perfect gas and ideal magneto-gasdynamic equation by using substitution principle. Murata [12] has obtained an exact solution to the system of PDEs that governs the one dimensional, unsteady motion of a perfect gas with strong shocks. Singh, Husain and Singh [13] used the method of Lie group transformation to obtain an approximate analytical solution to the system of first order nonlinear partial differential equations that governs a compressible one-dimensional unsteady planer, cylindrically symmetric and spherically symmetric motion in a non-ideal gas, involving strong discontinuities. Later, Singh, Ram and Singh [14] refined the exact solution of the blast wave in a non-ideal medium with the use of an analytical approach, which had proved the predication of the blast waves propagating outwards from the eruption. Singh, Ram and Singh [15] gave the exact solution of planar and non-planar weak shock wave problem in compressible gas dynamics with generalized geometries. Siddiqui and Arora [16] calculated the exact solution of shock wave problem with spherical symmetry in a relaxing gas using similarity transformation. 
Chaudhary and Singh [17] reported the exact solution of system of differential equations governing the propagation of weak shock waves in a compressible one dimensional non-ideal adiabatic gas flow. In the present study, we consider a $3 \times 3$ system of PDEs that govern one-dimensional non-steady planar and radially symmetric flow of a van der Waals gas (Real gas) with small solid dust-laden particles in the absence of viscosity. In this context, we assumed that the undisturbed gas density ahead of the shock front varies as a power law of the distance from the center of disturbance. An efficient analytical approach is used to determine a closed form exact solution of flow variables that exhibits position time dependence influenced by van der Waals excluded volume and small solid dust-laden particles. Finally, an analytical expression for the total energy influenced by van der Waals excluded volume in the presence of dust-laden particles is derived.

\section{BASIC EQUATIONS AND PROBLEM FORMULATION}

We consider disturbances for the one-dimensional compressible flow of a Van der Waals gas with dust-laden particles whose equation of state (Pai [18], Pai, Menon and Fan [19], Chadha and Jena [20, 21]) is governed by

$$
p=\frac{\left(1-k_{p}\right)}{(1-Z)(1-b \rho)} \rho R T \text {, }
$$

where $p$ is the pressure of the gas particle, $\rho$ is the density,

$R$ is the specific gas constant and $T$ is the temperature of the gas and that of the solid particles. The entity $Z=V_{s p} / V_{g}$ denotes the volume fraction where $V_{s p}$ is the volumetric extension of the solid particles and $V_{g}$ is the total volume of the mixture. The ratio of the total mass of the solid particles $\left(m_{s p}\right)$ and total mass of the mixture $\left(m_{g}\right)$ gives mass concentration of the solid particles in the mixture and it is denoted by $k_{p}$ i.e. $k_{p}=m_{s p} / m_{g}$. Here, $\bar{b}=b\left(1-k_{p}\right)$ where $b$ is the Van der Waals excluded volume that lies in the interval $0.9 \times 10^{-3} \leq b \leq 1.1 \times 10^{-3}$. The relation between the entities $Z$ and $k_{p}$ are given by the expression $Z=\theta \rho$, where $\theta=k_{p} / \rho_{s p}$ and $\rho_{s p}$ represents the species density of the solid particles.

For the current investigation, the governing equations are made to formulate on account of the following assumptions:

(i) The dusty particles are of uniform size, spherical, amorphous and less than 5\% of the entire volume. Each dust particle has the same temperature and their specific heat is constant. The particles are permanent and collisions between particles of different sizes will be neglected.

(ii) The particles are evenly distributed over the cross section of the duct and in comparison of the cross-sectional dimensions of the duct; the average spacing and size of the dust particles are small. Boundary layer effects and heat transfer with the duct walls will be neglected. (iii) The effect of the gas particles is first appears in view of the particles and then distributed over the rest of the gas by mixing. No external forces applied on the mixture of the gas and dusty particles, and the pressure is not influenced by the dust particles.

The basic equations governing a planar $(m=0)$ cylindrically symmetric $(m=1)$ or a spherically symmetric $(m=2)$ flow of a non-ideal gas with dust particles whose equation of state is given by Eq. (1), can be written in the following form [18-21].

$$
\begin{aligned}
& \frac{\partial \rho}{\partial t}+u \frac{\partial \rho}{\partial x}+\rho \frac{\partial u}{\partial x}+\frac{m \rho u}{x}=0 \\
& \frac{\partial u}{\partial t}+u \frac{\partial u}{\partial x}+\frac{1}{\rho} \frac{\partial p}{\partial x}=0 \\
& \frac{\partial p}{\partial t}+u \frac{\partial p}{\partial x}+\rho a^{2}\left(\frac{\partial u}{\partial x}+\frac{m u}{x}\right)=0
\end{aligned}
$$

where

$a=\left[\frac{\left(\Gamma-\alpha_{2} \rho^{2}\right) p}{\left(1-\alpha_{1} \rho+\alpha_{2} \rho^{2}\right) \rho}\right]^{\frac{1}{2}}$

where $a$ is the equilibrium speed of sound in the mixture with $\alpha_{1}=\theta+\bar{b}$ and $\alpha_{2}=\theta \bar{b}$.

$\Gamma$ is the Gruensien coefficient defined as $\Gamma=\gamma(1+\lambda \beta) /(1+\lambda \beta \gamma) \quad, \quad \lambda=k_{p} /\left(1-k_{p}\right)$, $\beta=c_{s p} / c_{p}, \gamma=c_{p} / c_{v}$ where $c_{s p}$ is the specific heat of solid particles, $C_{p}$ is the specific heat of the gas at constant pressure, and $C_{v}$ is the specific heat of the gas at constant volume.

Let, it is assumed that the position of the shock front from center of disturbance be given by $R=R(t)$ as a function of time $t$ with shock speed $\frac{d R}{d t}=V$ propagating into the inhomogeneous medium given by $u_{0}=0, p_{0}=$ constant and $\rho=\rho_{0}(x)$.

Across the shock front $R=R(t)$, the following Rankine-Hugoniot jump conditions are satisfied:

$\rho=\frac{\Gamma-1+2 \alpha_{1} \rho_{0}}{4 \alpha_{2} \rho_{0}{ }^{2}}\left\{1 \pm \sqrt{1-\frac{8(\Gamma+1) \alpha_{2} \rho_{0}{ }^{2}}{\left(\Gamma-1+2 \alpha_{1} \rho_{0}\right)^{2}}}\right\} \rho_{0}$

$u=\left[1-\frac{4 \alpha_{2} \rho_{0}^{2}}{\Gamma-1+2 \alpha_{1} \rho_{0}}\left\{1 \pm \sqrt{1-\frac{8(\Gamma+1) \alpha_{2} \rho_{0}^{2}}{\left(\Gamma-1+2 \alpha_{1} \rho_{0}\right)^{2}}}\right\}^{-1}\right] V$

(7) 


$$
p=\left[1-\frac{4 \alpha_{2} \rho_{0}^{2}}{\Gamma-1+2 \alpha_{1} \rho_{0}}\left\{1 \pm \sqrt{1-\frac{8(\Gamma+1) \alpha_{2} \rho_{0}^{2}}{\left(\Gamma-1+2 \alpha_{1} \rho_{0}\right)^{2}}}\right\}^{-1}\right] \rho_{0} V^{2}
$$$$
\zeta=\frac{2\left(1-\alpha_{1} \rho_{0} \mu_{2}+\alpha_{2} \rho_{0}^{2} \mu_{2}^{2}\right)}{(m+1)(2-\xi)\left(\Gamma-\alpha_{2} \rho_{0}^{2} \mu_{2}^{2}\right)+(3 m+1) \xi\left(1-\alpha_{1} \rho_{0} \mu_{2}+\alpha_{2} \rho_{0}^{2} \mu_{2}^{2}\right)}
$$

In the present study, the undisturbed density $\rho_{0}$ is taken to vary according to the power law of the radius of the shock front $R$ after the disturbance and is given as

$$
\rho_{0}=\rho_{v} R^{\eta}
$$

where $v$ and $\eta$ are constants. The constant $\eta$ is to be determined in further analysis.

\section{EXACT SOLUTION OF THE BLAST WAVE PROBLEM}

We construct an expression for the pressure in the flow field satisfying the Rankine-Hugoniot relations (7) - (9) as

$$
\begin{aligned}
& p=\left[\frac{4 \alpha_{2} \rho_{0}{ }^{2}}{\left(\Gamma-1+2 \alpha_{1} \rho_{0}\right) \pm \sqrt{\left(\Gamma-1+2 \alpha_{1} \rho_{0}\right)^{2}-8(\Gamma+1) \alpha_{2} \rho_{0}^{2}}-4 \alpha_{2} \rho_{0}{ }^{2}}\right] \\
& \rho u^{2}
\end{aligned}
$$

Using Eq. (10), Eq. (3) and (4) are converted in the form of following set of equations:

$$
\begin{aligned}
& \frac{\partial u}{\partial t}+u \frac{\partial u}{\partial x}+\frac{1}{\mu_{1}}\left(\frac{u^{2}}{\rho} \frac{\partial \rho}{\partial x}+2 u \frac{\partial u}{\partial x}\right)=0 \\
& \frac{\partial u}{\partial t}+u \frac{\partial u}{\partial x}+\frac{1}{2}\left\{\frac{\Gamma-1+\alpha_{1} \rho_{0} \mu_{2}-2 \alpha_{2} \rho_{0}^{2} \mu_{2}^{2}}{1-\alpha_{1} \rho_{0} \mu_{2}+\alpha_{2} \rho_{0}^{2} \mu_{2}^{2}}\right\} u\left(\frac{\partial u}{\partial x}+\frac{m u}{x}\right)=0
\end{aligned}
$$

where $\mu_{1}$ and $\mu_{2}$ are given as

$$
\begin{aligned}
& \mu_{1}=\frac{\left(\Gamma-1+2 \alpha_{1} \rho_{0}\right) \pm \sqrt{\left(\Gamma-1+2 \alpha_{1} \rho_{0}\right)^{2}-8(\Gamma+1) \alpha_{2} \rho_{0}^{2}}-4 \alpha_{2} \rho_{0}^{2}}{4 \alpha_{2} \rho_{0}^{2}}, \\
& \mu_{2}=\frac{\left(\Gamma-1+2 \alpha_{1} \rho_{0}\right) \pm \sqrt{\left(\Gamma-1+2 \alpha_{1} \rho_{0}\right)^{2}-8(\Gamma+1) \alpha_{2} \rho_{0}^{2}}}{4 \alpha_{2} \rho_{0}^{2}} .
\end{aligned}
$$

On combining Eqs. (11) and (12) and after integrating the resulting equation we obtained,

$$
\rho u^{2-\xi} x^{-m \xi}=f(t),
$$

where $f(t)$ is a function of time and $\xi$ is given by

$$
\xi=\frac{1}{2} \frac{\mu_{1}\left(\Gamma-1+\alpha_{1} \rho_{0} \mu_{2}-2 \alpha_{2} \rho_{0}^{2} \mu_{2}^{2}\right)}{1-\alpha_{1} \rho_{0} \mu_{2}+\alpha_{2} \rho_{0}^{2} \mu_{2}^{2}}
$$

In view of Eq. (13), Eq. (1) can be expressed in the following form:

$$
\frac{(2-\xi)}{u} \frac{\partial u}{\partial t}+(1-\xi) \frac{\partial u}{\partial x}-\frac{m(\xi+1) u}{x}-\frac{1}{f} \frac{d f}{d t}=0 .
$$

On solving Eq. (15) together with Eq. (12), we get

$$
u=-\zeta \frac{x}{f} \frac{d f}{d t}
$$

where the constant $\zeta$ is given by
Using Eq.(16), the expression for $f(t)$ can be written as

$$
f(t)=f_{0} t^{-\kappa} \text {, }
$$

where $f_{0}$ is arbitrary constant and $\kappa$ is given as

$$
\kappa=\frac{\xi-2}{(\xi-1) \zeta+m(\xi+1) \zeta-1} \text {. }
$$

With the help of Rankine-Hugoniot condition (7) and in view of Eq. (16), the analytical expression for the radius of the shock front is given as

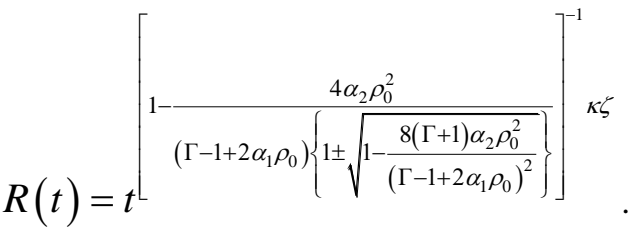

Using Rankine-Hugoniot condition (6) and in view of Eq. (9) we may obtained the value of the constant $\eta$, given as

$\eta=\frac{(2 m \xi+m-1) \mu_{2}-\{(m+1)+\xi(m-1)\}}{\mu_{2}}$.

Therefore, the exact solution to the blast wave problem governing by Eqs. (2)-(4) subject to the Rankine-Hugoniot jump conditions (6)-(8) is given as

$\rho=f_{0}(\kappa \zeta)^{\xi-2} \frac{x^{(m+1) \xi-2}}{t^{\xi+\kappa-2}}$

$u=\kappa \zeta \frac{x}{t}$

$p=\left[\frac{4 \alpha_{2} \rho_{0}^{2} f_{0}(\kappa \zeta)^{\xi}}{\left(\Gamma-1+2 \alpha_{1} \rho_{0}\right) \pm \sqrt{\left(\Gamma-1+2 \alpha_{1} \rho_{0}\right)^{2}-8(\Gamma+1) \alpha_{2} \rho_{0}^{2}}-4 \alpha_{2} \rho_{0}^{2}}\right] \frac{x^{(m+1) \xi}}{t^{\xi+\kappa}}$

The total amount of energy $E$ carried by the blast wave is equal to the sum of the kinetic and internal energy of the gas. The expression of the total energy for disturbances in a non-ideal medium under the influence of dust particles is given as

$$
E=4 \pi \int_{0}^{R}\left\{\frac{1}{2} \rho u^{2}+\frac{(1-Z)(1-\stackrel{G}{b} \rho)}{\left(1-K_{p}\right)} p\right\} x^{m} d x
$$

On substituting the values of flow variables from Eq. (22)-(24) in Eq. (25) we have

$E=A t$ $\kappa \zeta(m+1)(\xi+1) \mu_{2}-(\xi+\kappa) \mu_{1}$

where the constant $A$ is given by 


$$
A=\frac{2 \pi f_{0}}{(m+1)(\xi+1)}\left[\frac{2}{(m+1)\left(\frac{\Gamma-\alpha_{2} \rho_{0}^{2} \mu_{2}^{2}}{1-\alpha_{1} \rho_{0} \mu_{2}+\alpha_{2} \rho_{0}^{2} \mu_{2}^{2}}\right)-(m-1)}\right]^{\xi}\left[1+\frac{2(1-Z)}{\mu_{1}\left(1-k_{p}\right)}\left(1-\stackrel{\odot}{b} \rho_{0} \mu_{2}\right)\right]
$$

\section{RESULT AND DISCUSSION}

In the present investigation, we have employed an efficient analytical technique to determine the exact solution to a system of non-homogeneous quasilinear hyperbolic PDEs involving blast waves, which governs an inviscid one-dimensional unsteady planar and non-planar flow of a van der Walls gas affected by small solid dust-laden particles. The values of flow parameters which exhibit space time dependence are determined by analytical computations. The influence of van der Walls excluded volume and dust particles on the flow variables is noticeable from Eq. (22)-(24). It is worth mentioning that in the absence of non-ideal parameter $(b=0)$ and dust particles $(\theta=0)$, the results obtained shows a closer agreement with the earlier results carried out by various approaches in different material media. In addition, in view of Eq. (26), it has been observed that the total energy carried by the blast wave in a non-ideal medium under the influence of van der Waals excluded volume and, mass and volume concentration of small solid dust particles varies with respect to time. The reported analytical results exploring significantly that how the flow parameters as well as the total energy carried by the blast waves is influenced by dust-laden particles and parameter of non-idealness.

\section{CONCLUSIONS}

In this article, an efficient analytical technique has used to determine the exact solution to a non-homogeneous system of quasilinear hyperbolic PDEs involving strong discontinuities, which governs an inviscid one-dimensional unsteady planar, cylindrically symmetric and spherically symmetric flow of a real gas affected by small solid dust particles. The analytical solution of the flow parameters density, velocity and pressure has presented in the form of the power in the distance and the time. Consequently, an expression is derived for the total energy, in which the influence of van der Waals excluded volume and, mass and volume concentration of small solid dust particles is significant.

\section{ACKNOWLEDGMENT}

The authors are thankful to the referees for making certain points more explicit.

\section{REFERENCES}

1. G. I. Taylor, "The formation of a blast wave by a very intense explosion. I. theoretical discussion," Proc. Roy. Soc. London. Ser. A, Math. and Phys. Sci., vol. 201, 1950, pp. 159-174.

2. G. I. Taylor, "The formation of a blast wave by a very intense explosion. II. the atomic explosion of 1945," Proc. Roy. Soc. London. Ser. A, Math. and Phys. Sci., vol. 201, 1950, pp. 175-186.

3. A. Sakurai, "On the propagation and structure of the blast wave, I", J. Phys. Soc. Jpn., vol. 8, 1953, pp. 662-665.

4. A. Sakurai, "On the propagation and structure of a blast wave, II", J. Phys. Soc. Jpn., vol. 9, 1954, pp. 256-266.
5. M. H. Rogers, "Analytic solutions for the Blast-waves problem with an atmosphere of varying density," Astrophys. J., vol. 125, 1957, pp. 478-493.

6. G. G. Bach, J. H. S. Lee, "An analytical solution for blast waves,' AIAA, vol. 8, 1970, pp. 271-275.

7. S. A. Poslavskii, "A new class of exact solutions with shock waves in gas dynamics,” PMM, vol. 49, 1985, pp. 752-757.

8. V. D. Sharma, R. Ram, P. L. Sachdev, "An analytical solution to the problem of decaying shock wave,” J. Fluid Mech., vol. 185, 1987, pp. 153-170.

9. Z. Zhuang, D. Xue, Z. Chen, X. Jiang, "The eruption of a high pressure cylindrical heavy gas cloud ," Canadian J. Phys., vol. 91, 2013, pp. 850-854.

10. F. Oliveri, M. P. Speciale, "Exact solutions to the unsteady equations of perfect gases through Lie group analysis and substitution principles,” Int. J. Nonlinear Mech., vol. 37, 2002, pp. 257-274.

11. F. Oliveri, M. P. Speciale, "Exact solutions to the ideal magnetogasdynamics equations through Lie group analysis and substitution principles," J. Phys. A, vol. 38, 2005, pp. 8803-8820.

12. S. Murata, "New exact solution of the Blast wave problem in gas dynamics," Chaos, Solitons and Fractals, vol. 28, 2006, pp. 327-330.

13. L. P. Singh, Akmal Husain, M. Singh, "An approximate analytical solution of imploding strong shocks in a non-ideal gas through lie group analysis," Chin. Phys. Lett., vol. 27, 2010, pp. 014702(1)-014702(4).

14. L.P. Singh, S. D. Ram, D. B. Singh, "Analytical solution of the blast wave problem in a non-ideal gas," Chin. Phys. Lett., vol. 28, 2011, pp. 114303(1)-114303(3).

15. L.P. Singh, S.D. Ram, D.B. Singh, "Exact solution of planar and non-planar weak shock wave problem in dynamics," Chaos, Solitons and fractals, vol. 44, 2011, pp. 964-967.

16. M. J. Siddiqui, R. Arora, "An exact similarity solution for spherical shocks in a relaxing gas," Natl. Acad. Sci. Lett., vol. 38, 2015, pp. 433-435.

17. J. P. Chaudhary, L. P. Singh, "Exact solution of the weak shock wave in non-ideal gas," Int. J. Appl. Comput. Math, vol. 4, 2018, pp. 136

18. S.I. Pai, Two Phase Flows, Vieweg Verlag, Braunschweig, 1977.

19. S.I. Pai, S. Menon, Z.Q. Fan, "Similarity solution of a strong shock wave propagating in a mixture of a gas and dusty particles," Int. J. Eng. Sci., vol. 18, 1980, pp. 1365-1373.

20. M. Chadha, J. Jena, "Self-similar solutions and converging shocks in a non-ideal gas with dust particles," Int. J. Nonlinear Mech., vol. 65, 2014, pp. 164-172.

21. M. Chadha, J. Jena, "Singular surface and steeping of waves in a non-ideal gas with dust particles," Comp. Appl. Math., vol. 34, 2015, pp. 729-739.

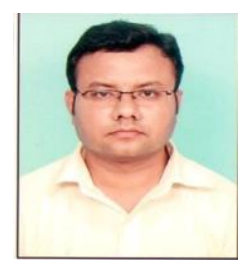

\section{AUTHORS PROFILE}

Dr. Akmal Husain pursed Master of Science from Purvanchal University in year 2005. He is completed his Ph.D. from IIT (BHU), Varanasi and currently working as an Assistant Professor (SG) in Department of Mathematics, SoE, University of Petroleum and Energy Studies, Dehradun since 2013. He has published 14 research papers in reputed international journals and conferences. His main research work focuses on quasilinear systems of partial differential equations, conservation laws, nonlinear waves in gas-dynamics, Lie group invariance for solutions of PDEs, and two-phase flows. He has 9 years of teaching experience and 6 years of research experience.

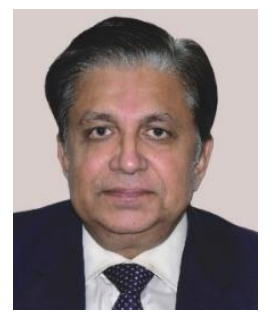

Prof. V. K. Singh is working as a Professor in the Department of Applied Sciences, Institute of Engineering and Technology, Lucknow. Presently, He is working as a vice-chancellor of Deen Dayal Upadhyaya Gorakhpur University, Gorakhpur. His research interest focuses on propagation of non-linear waves in gaseous media. $\mathrm{He}$ has

published more than 30 research papers in reputed international journals and conferences. 


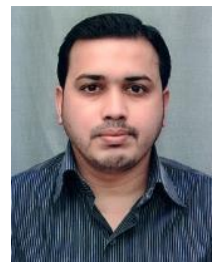

Mr. Syed Aftab Haider pursed M.A. in Mathematics from MJP Rohilkhand University in year 2005. He qualified CSIR-NET (Mathematical Sciences) in year 2013 and GATE (Mathematics) in year 2014. He is currently pursuing Ph.D. from AKTU Lucknow under the supervision of Dr. V. K. Singh, Department of Applied Sciences, Institute of Engineering and Technology, Lucknow-226021, India and co-supervision of Dr. Akmal Husain, Department of Mathematics, University of

Petroleum and Energy Studies, Dehradun-248007, India. In addition, He is currently working as an assistant professor in the Department of Mathematics, Shia P.G. College, Lucknow. 